\title{
1 Warum Ethik managen?
}

Über Management, Medizin und Ethik. Das Health-Care-Team. Der Zusammenhang zwischen Managementkompetenz, Führungsstärke und Ethik-Kompetenz. Warum Ethikinfrastrukturen und Ethik-Kompetenz nicht dem Zufall überlassen werden sollten. Gestaltung ethischer Diskurse und Konzepte durch das Management. Vertrauensbildung und Unternehmensstrategie durch verbindliche Ethik.

\subsection{Management „macht“ Medizin}

„Management“ im Krankenhaus ist mehr als die frühere „Verwaltung“. Kaufmännische, ärztliche, pflegerische und weitere Ebenen gehören dazu. Management im Gesundheitswesen verfolgt letztlich dieselben Ziele wie die Gesundheitspolitik und die Medizin. Management, Medizin und Gesundheitspolitik erfahren ihre Legitimation aus der Mehrung von „Gesundheit“. Und sie verlieren ihre Legitimation, wenn sie dieses Ziel verfehlen.

Management gestaltet Strukturen, Prozesse, Organisationen, Unternehmen und wird daran gemessen, ob die verfolgten Ziele wirtschaftlich und effizient erreicht werden. Mit seinen spezifischen Methoden und Kompetenzen dient es der Förderung, dem Einsatz und der Gestaltung all jener Berufsgruppen, Wissenschaften und Künste, die in der Lage sind, „Ge- 
sundheit" zu erhalten und wiederherzustellen, bei chronischen Krankheiten ein möglichst gutes Leben zu ermöglichen und das Leben zu verlängern, wo immer dies mit gutem Gewissen möglich ist. Auch die bestmögliche Begleitung Sterbender gehört zu diesen Aufgaben. Peter F. Drucker hat das Krankenhaus als eine der komplexesten Organisationen mit den höchsten Herausforderungen für das Management bezeichnet. Die vielfältigen Anforderungen an Qualität und Ethik der Patientenversorgung tragen hierzu wesentlich bei (s. Abb. 1).

In diesem Buch wird der Begriff „Medizin“ im erweiterten Sinn verwendet. In diesem Verständnis umfasst er auch die Pflege und die nicht-ärztlichen Therapeuten. Es ist wichtig darauf hinzuweisen, dass „die Medizin“ über keine abgeschlossene Identität verfügt. Der Begriff ist im ständigen Wandel und in seiner Grenzziehung stets auch von Interessen abhängig.

Management im Gesundheitswesen „macht Medizin“. Freilich nicht wie die Ärzte und die vielen Berufe, die direkt mit Patienten arbeiten, sondern indirekt, indem es die Arbeitsbedingungen und Ressourcen der unmittelbaren Gesundheitsberufe auf Unternehmensebene gestaltet. Damit steht es auch mit in der medizinischen Verantwortung. Es kann sich nicht in die kaufmännische Ecke zurückziehen und sollte auch aus dieser befreit werden. Management muss etwas von der Medizin verstehen, da die

\section{Herausforderung Krankenhausmanagement}

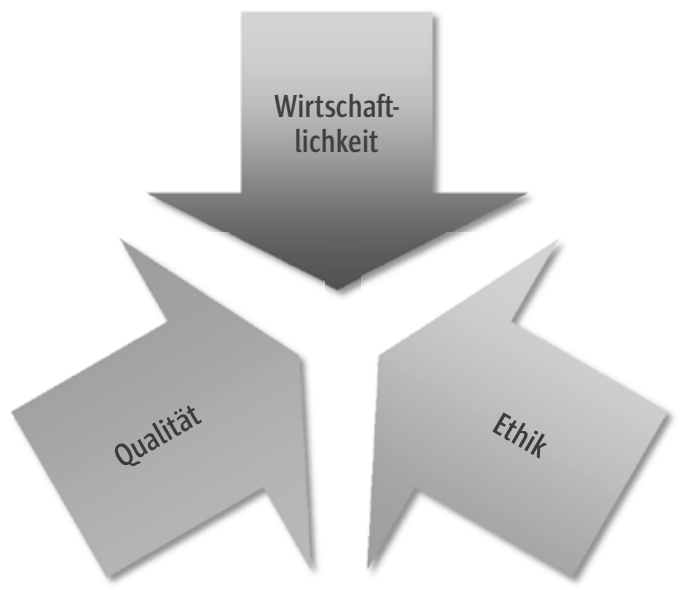

Abb. 1 Herausforderung Krankenhausmanagement.

Peter F. Drucker hat das Krankenhaus als eine der komplexesten Organisationen mit den höchsten Herausforderungen für das Management bezeichnet. Insbesondere die vielfältigen Anforderungen an Qualität und Ethik der Patientenversorgung tragen hierzu bei. 
Unternehmensziele nicht vom Ziel guter medizinischer Versorgung gelöst werden dürfen. Es ist Teil des „Health Care Teams“ und sollte in der Praxis auch so wahrgenommen werden.

\title{
1.2 Das Management als Teil des Healthcare Teams
}

Im Zentrum der individuellen und gesellschaftlichen Sorge um die Gesundheit steht zweifellos die Medizin, ohne die ein Gesundheitssystem und auch ein Krankenhaus keinen Sinn machen. Die Inhalte, Entwicklungen, Denkweisen und Potenziale der Medizin müssen das Management interessieren, weil sie vielfältige Konsequenzen haben für die Institutionen und Unternehmen. Nicht zuletzt sind sie grundlegende für das Angebot an Gesundheitsdienstleistungen und die Nachfrage der Patienten oder Kunden.

\begin{abstract}
Healthcare team
Für diesen englischen Begriff gibt es leider kein deutsches Wort. Wo es kein Wort gibt, gibt es auch keine reale Entsprechung. In deutschen Krankenhäusern ist der Teamgedanke am ehesten in der Intensivmedizin, Palliativmedizin und in den operativen Fächern oder der Psychiatrie realisiert. Die Managementberufe werden bislang aber meist nicht zum „Team“ gezählt. Vielfach werden sie „der Verwaltung“ zugeordnet und mehr oder weniger als fremd bis feindlich betrachtet. Je stärker das Management in die Abläufe der unmittelbaren Patientenversorgung eingreift umso wichtiger wird die Überwindung dieser Trennung.
\end{abstract}

Die Medizin befindet sich in einem kontinuierlichen Veränderungsprozess mit teilweise dramatischen Konsequenzen für Gesunde, Kranke, Nochnicht Kranke und letztlich für die gesamte Kultur und Gesellschaft. Die sich rasend schnell entwickelnde Grundlagenforschung im Bereich der Molekulargenetik, der Neurowissenschaften, der Nanoforschung und der Informationstechnologie ist aktuell dabei, ein völlig neues Konzept von „Medizin“ hervorzubringen, so dass unter anderen die Frage zu beantworten ist, was denn als Medizin gelten soll und was das Krankenhaus der Zukunft ausmachen wird. Auch die Psychotherapie, die Therapie- und Pflegewissenschaften gehören zu diesem Kern. Während sich all diese Professionen, Disziplinen und Wissenschaften direkt auf gesunde und kranke Individuen beziehen, richten sich all jene als „Public Health“ (früher auch „Sozialmedizin“) bezeichneten Maßnahmen auf Lebensbedingungen, Umwelten, Systeme und Verhaltensweisen. In mancher Hinsicht ist Management mit „Public Health“ verwandt, denn anders als beim be- 
handelnden Arzt ist der „Gegenstand“ der Maßnahmen ein Kollektiv. Wenn Management auf Wirtschaftlichkeit verpflichtet ist, so geht es auch- jedenfalls im öffentlichen Bereich- um das Ziel, als begrenzt geltende Ressourcen so einzusetzen, dass die Gesundheit der Bevölkerung davon bestmöglich profitiert.

Management im Gesundheitswesen organisiert, steuert und strukturiert eine wachsende Vielfalt von Gesundheitspraktiken. Es sorgt für die Bereitstellung geeigneten Personals, für effektive Teamarbeit, für die Cestaltung der Prozesse und Abläufe. Es stellt erforderliche Ressourcen einschließlich Wissen und Informationen bereit. Es kümmert sich um Finanzierungen, um Wirtschaftlichkeit, Gewinne und - im privaten Sektor-Profit. Dazu gestaltet es Organisationen, Institutionen, Unternehmen. Diese wiederum sind Teil eines politisch und juristisch gestalteten „Systems“", das Verantwortlichkeiten, Zielvorgaben und Finanzierungsmuster vorgibt. Alle drei Ebenen, die Mikroebene der persönlichen Betreuung und Versorgung, die Mesoebene der Unternehmen und die Makroebene aus Politik, Recht und Kultur müssen vom Management berücksichtigt und integriert werden. Ohne die Mikroebene jedoch, ohne die direkte medizinische, therapeutische, pflegerische und pädagogische Arbeit am einzelnen Menschen machen Management und Politik jedoch keinen Sinn.

Aus der grundlegenden, wenn auch in der Gegenwart noch wenig entwickelten Verbindung zwischen der praktizierten Medizin und dem Management ergibt sich, da es um Gesundheit und Krankheit und damit das Leben von Menschen geht, eine geteilte hohe Verantwortung. Die Organisation und „Führung“ medizinischer und pflegerischer Prozesse nimmt direkten und indirekten Einfluss auf deren Qualität und Ergebnisse. Wie die Pflegenden und Ärzte am Krankenbett, in der Diagnostik oder am OPTisch ist auch das Management verantwortlich für die Qualität der Versorgungsleistungen. Gute Medizin und Pflege brauchen gute Organisationen, vernünftige Rechtsordnungen, adäquate Finanzierungen und letztendlich ein gutes Management. Das Management ist effektiv Teil des „Health-Care-Teams“.

Dieser Gedanke ist grundlegend für das vorliegende Buch. Die hohe Bedeutung des Managements sowohl für die wirtschaftliche Dimension der Gesundheitsversorgung als auch für das Leben von Menschen, ihre Lebensqualität und Lebensdauer, begründet auch die Notwendigkeit der Teilhabe des Managements (wie der Politik) an ethischen Reflexionen und Diskursen auf den verschiedenen Ebenen zu denen auch das Healthcare Team zählt. Darüber hinaus liegt hier die Grundlage für den Sinn eines Ethik-Managements, also einer praktischen Nutzung der Ressource Ethik für die gesamte Unternehmensentwicklung und -führung. 


\subsection{Management, Ethik und Sinngebung}

Auf der Mikroebene von Medizin und Pflege ist Ethik im Sinne von fest verankerten Wertmaßstäben und Prinzipien seit Alters her die Voraussetzung für das Vertrauen der Patienten und der Bevölkerung. Anders als zu den Zeiten des Hippokrates praktizieren die Gesundheitsberufe heute aber in Organisationen, Unternehmen, Rechts- und Finanzierungsordnungen, deren Leistungsfähigkeit ihrerseits mit volkswirtschaftlichen und politischen Faktoren eng verbunden ist. Diese Einbindungen machen nicht nur ein komplexes Gesundheitsmanagement erforderlich, sie schaffen auch dessen klassische Konfliktlage zwischen den Bedürfnissen des einzelnen Patienten, Klienten oder Kunden einerseits, und kollektiven Interessen andererseits. Auch die Erhaltungsinteressen der Organisationen (z.B. der Krankenhäuser) und die wirtschaftlichen Interessen der Gesundheitsberufe sowie der Gesellschaft insgesamt sind zu berücksichtigen. Management steht auch im Dialog mit der Politik, die über das Maß bereit zu stellender Ressourcen sowie die Prinzipien ihrer Aufbringung und Verteilung (Gerechtigkeit, Solidarität, Gleichheit usw.) zu entscheiden hat. Schließlich entscheiden auch die politischen Organe der Gesellschaft und der internationalen Gemeinschaft über Richtung und Grenzen des wissenschaftlichen Fortschritts.

Aus all dem folgt, dass Management nicht als Technik zu verstehen ist, sondern als eine Kunst, die auf Kompetenzen beruht und sich vieler Techniken bedient. Dazu muss es die verschiedenen Techniken und die sie erzeugenden Wissenschaften kritisch auf ihre Eignung und Wirkungen prüfen und bewerten. Die Wissenschaften stellen jedoch in der Regel nur „Objektwissen“ bereit, das heißt, sie beschreiben Zusammenhänge, Trends, Möglichkeiten der Beeinflussung, Gegenstände. Was die objektivierenden Wissenschaften nicht leisten können ist Antwort geben auf die schwierigsten Fragen, die das Management und die Politik herausfordern. Sie können nichts sagen über die zu verfolgenden Ziele, also darüber, was als Ziel und damit als „wertvoll“ erreicht werden soll. Um Ziele und Werte als Orientierung für Entscheidungen wird in Medizin, Management und Politik häufig gestritten. Ethik stellt Wissen, Techniken und Kompetenzen bereit, um solche Entscheidungen in transparenten Prozessen nach transparenten Kriterien und Begründungen zu fällen.

Aussagen über das, was erreicht werden soll sind zentraler Bestandteil von Führung. Diese wiederum gelingt nur, wenn das Ziel begründet und für gut gehalten wird. Dies ist eine Voraussetzung für Motivation und Identifikation mit dem Ziel. Und diese sind wiederum für das Ergebnis 


\begin{abstract}
von größter Bedeutung. Ethik-Kompetenz von Führungskräften bedeutet, den Sinn von Entscheidungen herauszuarbeiten und auch in der Situation von Konflikt und Dilemma entscheidungsfähig und führungskompetent zu bleiben.
\end{abstract}

\title{
1.4 Ethik als Kompetenz
}

Für die Praxis genügt es nicht, Wissen über philosophische, theologische oder medizinische Ethik zu haben. So wichtig solches Wissen ist, entscheidend ist die ethische Kompetenz. Damit ist die Fähigkeit gemeint, ethische Phänomene wahrzunehmen, in Sprache zu fassen und auf der Grundlage ethischer Expertise angemessen gestaltend einzugreifen. Wenn ein ethisches Problem oder - mehr noch - ein ethischer Konflikt vorliegt, kann dies zweierlei Situationen betreffen. Die erste liegt vor bei einem realen oder empfundenen „ethischen Fehlverhalten“, indem beispielsweise eine anerkannte Norm verletzt wird. Es kann sich um eine Verletzung der Schweigepflicht handeln, um eine Unterlassung der Sorgfaltspflicht oder um die Verschwendung von materiellen Ressourcen. Ein ethischer Konflikt liegt vor, wenn unterschiedliche Normen nicht miteinander vereinbar sind. Dies ist zum Beispiel der Fall, wenn ein Patient, für dessen Wohl man verantwortlich ist, in vollem Besitz seiner geistigen Kräfte („Autonomie“) etwas tut, das seiner Gesundheit und seinem Leben schadet. Ein ethischer Konflikt liegt auch vor, wenn man vor der Frage steht, ob die Notaufnahme wegen Überlastung geschlossen werden soll, während gleichzeitig eine hohe Belegung des Hauses für dessen wirtschaftliche Zukunft entscheidend ist.

Ethische Kompetenz besteht also in der Fähigkeit, mit Hilfe von Wissen die Wahrnehmung auf derlei Phänomene zu lenken, sie gedanklich und sprachlich zu fassen um eine begründet „gute“ Entscheidung treffen zu können. Ein ethisches Problem unterscheidet sich von einem technischen Problem dadurch, dass dies durch Sachwissen und Fakten allein nicht zu lösen ist, geht es doch um die Frage, was in einer Situation X getan werden soll. Ein Herzchirurg beispielsweise verfügt über die technische Kompetenz, ein Organ zu verpflanzen. Er verfügt auch über die Erfahrung, wie die Erfolgschancen einzuschätzen sind. Die Entscheidung aber, ob ein jüngerer oder ein älterer Patient das Herz bekommen sollte, kann er nicht besser treffen als jeder Laie. Ob ein Krankenhaus aus wirtschaftlichen Gründen „lukrative Patienten“ bevorzugt Termine vergeben soll enthält ebenfalls ethisches Konfliktpotenzial.

Sehr häufig sind ethische Phänomene daran erkennbar, dass die beteiligten Menschen emotional stark involviert sind. Sind eigene moralische 
Maßstäbe verletzt worden so bleiben emotionale Reaktionen nicht aus, weil die Selbstachtung im Spiel ist. Wer in solchen Situationen über die Fähigkeit zur Distanz verfügt, um die Frage danach zu stellen, was jeweils genau vor sich geht, der verfügte über einen Vorteil. Er kann die Reaktionen der Beteiligten klarer und schneller verstehen und respektvoll auf sie eingehen. So können Verständigungsprozesse im Sinne von Mediationen vorgenommen werden. Die eingesetzten Kriterien für die Beurteilung einer Situation und die darauf bauende Entscheidung werden klarer und verhandelbar. Die Erfahrung zeigt, dass in solchen Verfahren Einschätzungen geändert werden können und chronifizierte Teamkonflikte aufgelöst werden können.

\section{„Die mit dem Leichentuch wedeln“ versus „die Schreibtischmörder“}

Ein Fallbeispiel zum Thema „Ethischer Konflikt im Krankenhaus“ sowie zu Sinn und Nutzen von „Ethik-Strukturen“ und ethischer Kompetenz.

Das folgende Fallbeispiel ist keine Erfindung. Es beschreibt einen extremen Konflikt, der sich in einem kleineren Kreiskrankenhaus zugetragen hat. In diesem Ausmaß sind solche Konflikte zwar selten, die Grundstruktur liegt aber vielen täglichen Konflikten in deutschen Kliniken zu Grunde. Das wirtschaftliche Interesse eines Krankenhauses gerät in Konflikt mit dem moralischen Empfinden von Ärzten und Pflegenden, aber auch von Patienten, Angehörigen und Öffentlichkeit. Das verantwortliche Krankenhausmanagement, gemeinhin als „die Verwaltung“ bezeichnet, sieht sich moralisch an den Pranger gestellt. Dabei können auch von dieser Seite wichtige moralische Gründe angeführt werden. Das Krankenhaus muss durch Sicherung von Einnahmen seinen Erhalt sichern. Die Mitarbeiter und alle Leistungen müssen bezahlt werden. Die Kosten der Häuser müssen in Grenzen gehalten werden, weil die Zwangsbeiträge zur Krankenversicherung nicht beliebig gesteigert werden können.

Der Notruf einer Frau erreicht den Rettungsdienst. Ihre Mutter, die zu Besuch sei, sei bewusstlos und krampfend zusammengebrochen. Der Notarzt findet eine nicht ansprechbare 65-jährige Frau vor. Umgehend wird sie ins naheliegende kommunale Krankenhaus gefahren, das über eine "Stroke-Unit" zur Behandlung von Schlaganfällen verfügt. Auf dieser Station werden alle erforderlichen medizinischen Maßnahmen eingeleitet. Sie führen alsbald zu einer Stabilisierung der Situation, allerdings bleibt die Behandlung und Überwachung auf der Intensiveinheit weiter erforderlich. Am dritten stationären Tag erfährt das Patientenmanagement, dass keine ausreichende Versicherung vorliegt. Die Patientin stammt aus einem nicht zur Europäischen Union gehörenden Land und der Versicherungsnachweis ist offenbar nicht korrekt. Die kaufmännische Abteilung befürchtet, dass die Behandlungsrechnung von niemandem übernommen wird. Das Klinikum schreibt „rote Zahlen“. Das Management fordert deshalb die verantwortlichen Ärzte auf, die Patientin möglichst umgehend zu entlassen. Die Aufforderung löst Empörung bei Ärzten 
und Pflegenden aus. Ein typischer Konflikt zwischen "Schreibtischmörder" auf der einen Seite und „denen, die mit dem Leichentuch wedeln“ auf der anderen Seite bricht aus.

„Medizin“ und „die Verwaltung“ stehen sich feindselig gegenüber. Ärzte und Pflegende berufen sich auf medizinethische Grundsätze. Man müsse stets zum Wohle des Patienten handeln und dürfe nie wissentlich dem Patienten Schaden zufügen. Geld dürfe bei medizinischen Entscheidungen grundsätzlich keine Rolle spielen. „Die Verwaltung“ argumentiert mit der wirtschaftlichen Situation des Hauses. Alle Mitarbeiter würden doch auch erwarten, dass ihre Löhne bezahlt werden. „Die Politik“ sei nicht grenzenlos bereit, das wirtschaftliche Defizit dieses Hauses auszugleichen. Ein Verkauf an einen privaten Krankenhauskonzern könne unvermeidbar sein, wenn man nicht auf die Kosten der Behandlung und die möglichen Erlöse achten würde.

Den Mitarbeitern gegenüber argumentiert das kaufmännische Management mit der wirtschaftlichen Zukunft des Klinikums und mit deren Eigeninteressen an Lohn und an einem kommunalen Arbeitgeber. Die Mitarbeiter betrachten die Argumentation als unmoralisch.

Man einigt sich nach aggressivem Streit auf einen Kompromiss. Die Patientin wird nach fünf Tagen entlassen. Im PKW ihrer Tochter wird sie über mehrere hundert Kilometer in ihre Heimat gefahren, wo sie noch am Ankunftstag stirbt. Im Klinikum versucht man alles daran zu setzen, dass die Öffentlichkeit nicht über den Fall informiert wird. Seitens der Presse wurde eine Skandalisierung befürchtet die einen Imageschaden für das Klinikum zur Folge hätte, was wiederum zu wirtschaftliche Einbußen führen könnte.

Ein klinisches Ethik-Komitee war in diesem Fall nicht vorhanden, Organisationsethik war unbekannt, entsprechende Erfahrung und Kompetenzen fehlten. Hätte es diese Strukturen gegeben, so hätte der Konflikt entmoralisiert und deeskaliert werden können Die zwei in sich schlüssigen, aber miteinander unvereinbaren Argumentationen wären deutlich identifiziert worden: ein Dilemma. Im Idealfall wären auch Mitarbeiter des kaufmännischen Managements Teil des Komitees. Wahrscheinlich wäre man zu dem Ergebnis gekommen, dass beide Seiten durchaus ebenbürtige moralische Gründe anführen konnten. Letztlich handelt sich um einen Konflikt zwischen den Interessen eines Individuums (Patient) sowie seinem medizinischen „Anwalt“ und den Interessen der Gesellschaft an einem finanzierbaren Gesundheitssystem und ihrem „Anwalt" im kaufmännischen Management. Die grundlegende Akzeptanz beider Positionen hätte dem Konflikt den feindseligen Charakter nehmen können, so dass man gemeinsam nach der bestmöglichen Entscheidung gesucht hätte.

An Stelle einer in zwei feindliche Lager geteilten Mitarbeiterschaft wäre man dem Modell eines „Health-Care-Teams“ nähergekommen. Die Lösungen wären dann möglicherweise in Finanzierungsmodellen und Leitlinien für dergleichen Fälle zu finden gewesen. Vielleicht hätte man einen speziellen Fonds eingerichtet, eine Stiftung einbezogen oder geregelte Verfahren mit Krankenkassen oder politischen Trägern gesucht. Man hätte sogar die Presse in diese Konflikt- 
lage einbeziehen können und in der regionalen Bevölkerung einen Prozess der Meinungsbildung über medizinische Versorgungsgrundsätze und deren Finanzierung angestoßen.

\subsection{Was ist gemeint, wenn von „der Ethik“ die Rede ist?}

Das Wichtigste vorweg: Ethik ist nicht Moral, sondern die kritische Theorie der Moral. Moral sagt: „Man muss“. „Man soll“. „Das tut man nicht.“ usw. Ethik fragt: Wie ist denn das „man muss“ oder „man darf nicht“ begründet? Stimmen die Argumente? Muss das immer so sein?

Ethik - so verstanden - ist antidogmatisch. Ethik legt Wert auf den Gedanken, dass aus der (wissenschaftlichen) Beschreibung dessen was ist (Deskription) und dessen, wie es „funktioniert“ und „zusammenhängt“ (Analyse), nicht direkt abgeleitet werden kann, was man tun soll. Ethik fragt, „was soll ich tun?“, „was ist gut?“ Warum ist etwas „gut“ oder „schlecht"?

Wer sich mit „der Ethik“ befasst macht die Erfahrung, dass auch nach Jahrzehnte langem Studium sich keine unumstrittene Klarheit einstellt. Dennoch wird dieses Studium leicht als persönlicher Gewinn empfunden werden können.

Es gibt nicht „die Ethik“. Sicher ist, dass es den Begriff Ethik gibt und dass darunter ein hoch komplexes, nicht eindeutig definierbares Konglomerat von Denkweisen, Texten und Institutionen verstanden wird.

Der Begriff „Ethik“ gehört zu jenen sprachlichen Gebilden, von denen Nietzsche sagt, sie hätten sich von ihrem Gegenstand gelöst und würden ein (verwirrendes) Eigenleben führen. Damit sind auch einige Gefahren verbunden, weil die Rede von der Ethik missbraucht werden kann für Interessen der Macht.

Es ist wichtig zu beachten, dass immer dann, wenn die Formulierung „aus ethischer Perspektive“ oder „aus Sicht der Ethik“ benutzt wird, keineswegs eine einheitliche Position vertreten wird. Selten vertreten zwei „Ethiker“ die gleiche Meinung. Die Gemeinsamkeit liegt eher in der Art des Denkens und in der Nutzung gemeinsam verstehbarer Fachbegriffe. In der Regel geht es um eigene moralische Positionen der Autoren oder Sprecher, die „ethisch“ versucht werden zu begründen. In der Medizinethik geht es freilich um ein praktisches Ziel, nämlich um „gute Medizin“. 


\subsection{Ethik - eine Art des Denkens, Debattierens und Entscheidens}

Man könnte Ethik als eine Kunstform des Denkens bezeichnen, die darauf spezialisiert ist, sich mit Problemen moralischer Art zu befassen, also mit Normen und Werten im Sinne von Sitten und Regeln des gesellschaftlichen Verkehrs.

Die Besonderheit dieser Denkweise besteht darin, dass es letztendlich um die Frage geht, was getan werden soll. Diese Frage kann aus der Sicht einer Person, einer Institution, einer Profession oder einer ganzen Gesellschaft gestellt werden.

Mit Blick auf das Themenfeld Gesundheit ergeben sich beispielsweise folgende Fragen: Welche Ziele sollen bei der Behandlung eines Patienten verfolgt werden? Soll eine Klinik Schwangerschaftsabbrüche durchführen? Welchen Einfluss darf betriebswirtschaftliches Handeln auf medizinische Entscheidungen nehmen? Soll die Embryonenforschung gefördert oder verboten werden?

Ethisches Denken beinhaltet die Kunst, Fragen so zu stellen, dass unter verschiedenen möglichen Antworten der Blick darauf gerichtet wird, welche Antwort oder welches Ziel als gut angesehen werden kann.

Es geht also häufig darum, was gut ist, worin das Gute besteht und warum das Gute wirklich gut ist. Ist es zum Beispiel gut, Patientenverfügungen einzuführen, damit der mutmaßliche Wille eines nicht entscheidungsfähigen Patienten besser beachtet wird? Warum ist das so?

Die Frage nach dem, was als gut gelten soll und kann, die Postulierung von Werten, die zugleich Ziele zu bestimmen erlauben, ist die Vorstufe von Entscheidungen.

Wer eine Entscheidung trifft, macht im Sinne dieses Wortes einer Scheidung ein Ende, d.h. aus verschiedenen Alternativen wird eine Wahl getroffen. Der Entscheider betrachtet in der Regel seine Wahl als die beste, hat also eine Vorstellung davon, was gut, besser oder richtig ist.

Sich diese Wertmuster bewusst zu machen, bevor man anhand von Zahlen, Daten und Fakten glaubt, „rein sachliche“ Entscheidungen zu treffen, kann als angewandte Ethik bezeichnet werden.

Wer in diesem Sinne ethisch denkt, wird häufig die Erfahrung machen, dass es so genannte Zwickmühlen gibt, in der keine Entscheidung gut oder richtig ist. Solche Dilemmata können gefährlich werden, wenn sie Blockaden bewirken. Ethisches Denken lehrt die Kunst des Umgangs mit dem Dilemma oder anderen komplexen Situationen. 


\subsubsection{Ethik-Kompetenz als Fähigkeit gute Entscheidungen zu treffen}

Der Nutzen von Ethik für das Management im Krankenhaus und im Gesundheitswesen liegt in der Vermittlung von Kompetenzen. Diese schärfen das Bewusstsein für die moralische Dimension von Entscheidungen und die Macht moralischer Phänomene. „Gute“ Produkte und Dienstleistungen sind eine entscheidende Crundlage für jeden Unternehmenserfolg. Um „gut“ zu sein müssen sie auch auf „gute“ Weise erzeugt und erwirtschaftet werden. Umgekehrt kann die Verletzung moralischer Werte das Vertrauen von Kunden, Patienten, Einweisern und Öffentlichkeit erschüttern und die Existenz eines Unternehmens gefährden. Die Beispiele der Diesel-Abgasaffäre, zweifelhafter Kreditgeschäfte von Banken oder des Staffordshire-Skandals im Nationalen Gesundheitsdienst Großbritanniens (s.u.) zeigen dies in aller Deutlichkeit.

\section{Staffordshire Hospital: Leaders must exercise 'strong, ethical and patient- centred leadership in every organisation'}

Management, das vermeintlich nur an Zielen der Wirtschaftlichkeit und Effizienz orientiert ist und dabei auf eine starke ethische und patientenzentrierte Führungsqualität verzichtet, kann Katastrophen auslösen. Ein Beispiel dafür ist der in Deutschland wenig beachtete, im Vereinigten Königreich aber mit großer öffentlicher Aufmerksamkeit verfolgte „Staffordshire-Scandal“. Im MidStaffordshire Hospital sollen zwischen 2005 und 2008 bis zu 1.300 (!) Patienten an Unterversorgung zu Tode gekommen sein. Die Todesopfer stellen allerdings nur einen kleinen Teil der Opfer dar. Kosteneinsparungen und wirtschaftliche Zielvorgaben sowie deren Art und Weise der Umsetzung durch Führungskräfte wurden dafür verantwortlich gemacht. Als Reaktion wurden ein grundlegender Kulturwandel in den britischen Krankenhäusern und im gesamten National Health Service (NHS) eingefordert. Die von Robert Francis im Auftrag der britischen „Kings-Fund“-Stiftung durchgeführte Untersuchung schockierte die Öffentlichkeit Großbritanniens. Die dort gezogenen Lehren sind zweifellos auch für deutsche Führungskräfte im Gesundheitswesen von großer Bedeutung. Die folgenden Auszüge aus dem Bericht sind wörtlich übernommen.

"Leadership development is essential in promulgating an organisational culture in which patients' needs come first and the values of patient-centred care are communicated and understood at all levels, from the board to the ward.

It is the responsibility of every NHS organisation to give priority to developing its leaders - clinicians and managers - rather than expecting others to do it.

By the time, Stafford hospital's failings were exposed by regulators up to 1,200 patients had died needlessly. 
In the wards, people lay starving, thirsty and in soiled bedclothes, buzzers droning hopelessly as their cries for help went ignored. Some received the wrong medication; some, none at all.

To do this, they should:

- be visible and set an example

- listen to patients and staff

- understand their organisations in depth, at both an operational and strategic level

- think laterally across boundaries

- share leadership with all staff, and ensure that they feel valued, respected and supported

- encourage clinical engagement

- be able and willing to challenge others

A transformation of systems, leadership and organisational culture is needed throughout the NHS if the lessons of the Francis Inquiry into the shocking failings of care at Mid Staffordshire are to be learned and acted on.

The leadership of the NHS at a national level needs to create the conditions in which high standards of care are delivered consistently, setting clear goals and standards for improving quality and patient safety, and providing the means for staff to deliver these goals within available resources.

The quality of care provided by NHS organisations is first and foremost a corporate responsibility under the leadership of boards. As such, boards must demonstrate that they give sufficient priority to quality and patient safety - for example, by seeking and acting on patient feedback, hearing patient stories, reviewing and learning from complaints, taking time to listen to patients and their relatives, and acting on the results of staff surveys.

Clinical teams perform best when their leaders value and support staff, enable them to work as a team, ensure that the focus is on patient care, and create time to care."

\section{Management ist Praxis und Entscheiden}

Eine wichtige Voraussetzung um die Bedeutung ethischer Kompetenz für das Management im Gesundheitswesen zu verstehen ist ein Verständnis von Management als ein Tun, Handeln, Entscheiden (und nicht als „Objekt“). Dies ist keineswegs selbstverständlich. Die meisten deutschsprachigen Lehrbücher enthalten durchgängig „Objektwissen“, indem dort Gesundheitssysteme und deren Elemente wie Krankenversicherungen, Ärztekammer, Versorgungsstrukturen usw. beschrieben werden. Sie enthalten nützliches Wissen für Manager - das Management als Kunst des Handelns und Entscheidens kommt jedoch viel zu kurz. 
Die Rolle moralischer Bewertungen von Zielen, Mitteln, Strategien, Leistungen (Produkten) und Entscheidungen durch die Teams, die Kunden, Patienten und die Öffentlichkeit wird selten reflektiert. Ethik als Reflexionstheorie der Moral, als kritischer Gegenpol zur Moralisierung und als Kompetenz der Handlungs- und Entscheidungsfähigkeit in Situationen des Dilemmas erscheint in den Registern der Managementliteratur nur selten. Dabei sind diese Kompetenzen für das Management alles andere als entbehrlich. Sie gehören zu den „weichen Fähigkeiten“(„Soft Skills“), von denen Peter Drucker, der Initiator der Managementwissenschaften schreibt, sie seien ebenso wichtig wie die „harten Faktoren“.

\section{Hochleistung braucht Dissonanz: Zusammenführung von Notwendigkeit und Sinn im Kammerorchester Bremen}

Erfolgreiches Management setzt „Kultur“ ins Zentrum seines Konzepts. Diese bleibt nicht dem Zufall überlassen, sondern sie wird um wohl überlegte Werte und Normen herum entfaltet. Diese können durchaus Kontroversen und Auseinandersetzungen fördern, wenn sie denn in klare Entscheidungen überführt werden. Vorbildlich für ein handlungsorientiertes Management (auch) im Gesundheitswesen ist ein Werk über die Führung und Organisationskultur eines Kammerorchesters, das unter dem Titel „Hochleistung braucht Dissonanz“ die Herausforderungen an die Kompetenz von Führungskräften anschaulich behandelt. Orchester und ihre Dirigenten sind mehr als nur Metaphern für das Zusammenfügen der Tätigkeit vieler einzelner Menschen und deren Orientierung auf arbeitsteilig und gemeinsam zu verfolgende Ziele. So beruht der Erfolg der Deutschen Kammerphilharmonie Bremen, die zur Weltspitze der Orchester zählt, auf einer strategischen Zusammenführung von Notwendigkeit und Sinn, wobei die entscheidende Bedeutung bewusst reflektierter Normen und Werte in den Mittelpunkt des Managementkonzepts gestellt wird. „Letztlich ist es die Organisationskultur, die das Verhalten der Mitglieder des Hochleistungsteams zur Hochleistung bringt.“ Diese „kulturelle Perspektive zielt auf das Normen und Wertesystem."

\section{„Gute“ und „schlechte“ Ziele und Mittel: ihr moralischer „Impact“}

Im Krankenhaus, wo sehr unterschiedliche Wertesysteme integriert werden müssen, ohne ihre Eigenständigkeit aufgeben zu können, ist diese Fähigkeit von besonderer Bedeutung. Hier stoßen die Kulturen der Heilberufe (Ärzte, Pflegende, Therapeuten) auf die Kulturen der Betriebswirtschaft und die der Träger, seien sie nun religiöser, sozialer oder profitwirtschaftlicher Natur.

Führungspersonen sind Menschen, die Entscheidungen treffen und verantworten und andere Menschen für die Erfüllung spezifischer Aufgaben 
und Funktionen aussuchen, gewinnen und befähigen. Die Entscheidungen beziehen sich auf Ziele sowie auf die dafür geeigneten Mittel, Strategien und Ressourcen. Sowohl Ziele und Zwecke als auch die eingesetzten Mittel bedürfen einer moralischen Bewertung zum Zwecke der Rechtfertigung vor der Mitarbeiterschaft und der Öffentlichkeit. Viele Unternehmen aus verschiedensten Branchen stellen sich bewusst ihrer Unternehmensverantwortung, die unter dem Stichwort „Corporate Social Responsibility“ (CSR, vgl. Glossar) nicht nur den moralischen Wert ihrer Produkte, ihres Gewinnstrebens und sogar ihrer Gewinne zur Darstellung bringen, sondern auch die Moralität der Art und Weise, wie die Produkte hergestellt und Gewinne erwirtschaftet werden.

Auch wenn lediglich die Erwirtschaftung von Profit angestrebt wird, bedarf dies einer moralischen Legitimation vor der Öffentlichkeit. So wurde der kapitalistische Eigennutz von Adam Smith als Motor des Gemeinwohls charakterisiert und noch heute muss sich der globalisierte Kapitalismus vor der Weltöffentlichkeit kontrolliert legitimieren. Legitimationen erfolgen diskursiv, d.h. durch Sprache die auf der Basis empirischer Daten mit guten Argumenten überzeugen will.

Die vom Management verfolgten Ziele, die genutzten Mittel und die bedienten Interessen werden in höchstem Maße positiv oder negativ moralisch bewertet. Deshalb können sie sowohl Gegenstand gemeinschaftlicher und solidarischer Aktionen als auch heftigster Konflikte, Kämpfe ja Kriege sein. Positive oder negative Motivation, die sich oft in Begeisterung oder Empörung äußert, entscheiden über Unterstützung oder Widerstand (und Sabotage).

\section{Der bewusste Umgang mit den je spezifischen Normen- und Wertesys- temen ist Teil der Ethik-Kompetenz.}

Der moralische Charakter von Zielen und Interessen zeigt sich an der Unterscheidung von „guten“ und „schlechten“ Zielen und Mitteln, von akzeptablen und unakzeptablen Interessen, an Kriterien von Fairness, Wertschätzung und Erniedrigung. Das Ausmaß der Motivation von Führungskräften und Mitarbeitern ist erheblich von der moralischen Qualität bzw. Bewertung der Ziele und der zu ihrer Erreichung verfolgten Methoden/Konzepte abhängig. 


\section{Werte persönlich vertreten}

Im Idealfall sind wir mit Zielen und Mitteln identifiziert. Wir sind davon überzeugt, dass die Ziele „gut und richtig“ sind und dass die Mittel und Methoden, sie zu erreichen, „in Ordnung“ sind. Dazu müssen beide erkannt, bestimmt und moralisch bewertet werden. Dies geschieht in der Regel in analytischen, kommunikativen und diskursiven Prozessen, in denen Argumente ausgetauscht und entwickelt werden. Zahlen, Daten und so genannte Fakten unterstützen die Argumente, ersetzen sie aber nicht. Die Art und Weise, wie Entscheidungen vorbereitet, gefällt, kommuniziert und umgesetzt werden, prägen wesentlich die Unternehmenskultur. Diese durch das Management zu gestalten, kann mit Fug und Recht als Spitzenherausforderung betrachtet werden.

„Führungskräfte“ verkörpern die jeweiligen Ziele und Mittel in ihrer Person.

\section{Managementkompetenz und Ethik-Kompetenz}

Entscheidungsprozesse unterliegen ihrerseits Entscheidungen, nämlich darüber, wer am Prozess beteiligt wird, welche Themen gewählt oder ausgeschlossen werden, welche Expertise man zur Beratung zulässt, nach welchen Regeln die Entscheidung zustande kommt, welche Entscheidungskriterien zugelassen werden, was als Erfolgskriterium gelten soll und was nicht. Entscheidungsprozesse einschließlich der Entscheidungen über Entscheidungen gehören ins Aufgabenfeld des Managements. Da sie alle neben dem Sachaspekt einen moralischen Aspekt innehaben ist die Ethik-Kompetenz, die diesen systematisch reflektiert, für die Gestaltung einer guten Unternehmenskultur eine entscheidende Voraussetzung.

Das Erkennen und Bewerten moralischer Dimensionen von (nicht nur Unternehmens-)Zielen und Methoden ist bereits Ausdruck ethischer Kompetenz. Ethik-Kompetenz ist kein randständiges, schöngeistiges Blümchenwissen, sondern eine entscheidende, unverzichtbare argumentative „Waffenschmiede" für das Management.

Ethik-Kompetenz ist eine entscheidende, unverzichtbare argumentative „Waffenschmiede" für das Management.

In der Medizin, im Gesundheitswesen und auch in der „Gesundheitswirtschaft" sind es moralische Fragen, die die höchste Kompetenz aller Ent- 
scheider herausfordern. Auf der Ebene der Patientenversorgung sind dies die Behandlungsziele, zum Beispiel zwischen Lebenserhalt und Palliation. Auf der Managementebene (Mesoebene) geht es um die Herstellung der Vereinbarkeit von guter Medizin und betriebswirtschaftlichen Zielen. Auf der Makroebene (Politik, Recht, Internationale Entwicklung und Verantwortung) geht es um Fragen der Gerechtigkeit, der Solidarität, der Verantwortlichkeit („Wer soll für die Gesundheit des Einzelnen und der Gesellschaft verantwortlich sein?“).

Management ist weder ein Gegenstand noch eine Sache. Es ist ein „Tun und Handeln" von und mit Menschen, nach Zielen, auf der Basis von Interessen und unter der Wahl von Mitteln. Es lässt sich nicht auf „Technik“ reduzieren, da es in hohem Maße kommunikativ vermittelte Praxis ist. Ziele, Interessen und Mittel des Managements haben immer auch eine moralische Qualität, d.h. sie werden bewertet nach gut und schlecht, verantwortlich und unverantwortlich, gerecht und ungerecht, fair und unfair usw. Die Reflexion dieser moralischen Dimension nennen wir Ethik. Ethische Kompetenz ist die entscheidende Managementkompetenz!

In einer berühmten Tübinger Buchhandlung hing unlängst ein Poster mit dem Satz: „Ihr lest keine Lyrik? Seid ihr wahnsinnig?!“ Übertragen auf die Kompetenz des Managements heißt das: „Ihr nutzt die ,Ethik“ nicht! Seid Ihr wahnsinnig?!“

„Lancet- Kommission“ und Careum-Stiftung (Zürich) fordern Neuausrichtung der Ausbildung in den Gesundheitsberufen

Die Lancet Kommission (und unterstützend die Schweizer Careum Stiftung) hat eine globale soziale Bewegung zur Neuausrichtung der Ausbildung in den Gesundheitsberufen, zu denen auch das Management im Gesundheitswesen und damit auch im Krankenhaus zählt gefordert. Bei der Lancet-Kommission handelt es sich um eine mit internationalen Experten besetzte Kommission unter Schirmherrschaft des weltweit führenden Fachjournals für Medizin. Die herkömmlichen Ausbildungskonzepte betrachtet die Kommission als nicht länger geeignet, um den wachsenden Herausforderungen gerecht zu werden.

Die neuen Gesundheitsberufe sollen das „Silodenken“ zugunsten interprofessioneller Teambildung überwinden, über kritisches Urteilsvermögen verfügen, nach ethischen Gesichtspunkten handeln, Wissen umsetzen können, in lokal verantwortlichen und global vernetzte Teams arbeiten und an patienten- und bevölkerungsorientierten Gesundheitssystemen mitwirken.

Management sollte sich demnach nicht aus dem Fachgebiet der Betriebswirtschaftslehre allein definieren bzw. die Betriebswirtschaft sollte aus dem Zahlenghetto befreit werden und verstärkt ethische Kompetenz erwerben. Umgekehrt sollte auch die Ethik ihre akademischen Engführungen überwinden, sich in der gesellschaftlichen Praxis ansiedeln, sich mit sozialwissenschaftlicher 
Empirie und Theorie befassen und sich stärker mit ökonomischen Themen und Konzepten verbinden.

\subsubsection{Ethik: gesellschaftliche Institutionen, Strukturen und Praktiken}

„Die Ethik“ ist nicht nur eine Art des Denkens und Sprechens, sie ist auch ein Sammelbegriff für spezielle gesellschaftliche Institutionen und Praktiken. Sie gilt als eine akademische Disziplin.

Das ethische Denken und Sprechen hat sich seit der europäischen Antike als Teilgebiet der Philosophie und auch der Theologie an Akademien, Klöstern und Universitäten Institutionen geschaffen, die die Ethik pflegen, in Schriften niederlegen und weiterentwickeln.

Heute ist die Ethik eingebettet in internationale akademische Netzwerke mit weltweit zugängigen Datenbanken und Bibliotheken. Es gibt Ethik Institute als „Think Tanks“ und Forschungszentren. „Ethik“ kann studiert werden und die Berufsbezeichnung „Ethiker“ ist möglich. Es gibt Fachgesellschaften auf nationaler und internationaler Ebene, es gibt Fachkongresse und spezielle Publikationsmedien. Damit ist „die Ethik“ Teil des Wissenschaftsbetriebes.

Angesichts einer wachsenden Sensibilität gegenüber den Methoden, Risiken und Nebenwirkungen von Forschung im Allgemeinen und der Lebenswissenschaften im Besonderen sind Ethik-Einrichtungen zu Begleitern gesellschaftlich umstrittener Forschung und Technikentwicklung geworden.

Vielfach müssen Forschungsprojekte per Gesetz Ethik-Kommissionen zur Begutachtung vorgelegt werden. Dabei kommen international weitgehend allgemein anerkannte Ethik-Standards zur Anwendung, die wesentlich auf den Menschenrechten der Vereinten Nationen und der Deklaration von Helsinki des Weltärztebundes basieren (s. Kap. 2).

Es gibt zunehmend „Ethik Institutionen“ außerhalb der Hochschulen in verschiedensten Feldern des gesellschaftlichen Lebens. Ethik-Kommissionen und -Komitees prüfen und beraten, erarbeiten Leitlinien und liefern den Stoff für die Ausformulierung von Gesetzen.

In Deutschland gibt es einen Nationalen Ethikrat, der Präsident der USA hatte ein Ethical Advisory Board, Dänemark hat den Danish Council of Ethics, die UNESCO unterhält das International Bioethics Committee (IBC). Die konfessionellen Krankenhausverbände in Deutschland haben ihren Mitgliedskliniken die Einrichtung Klinischer Ethik-Komitees empfohlen. 
Wenn seit Aristoteles Ethik als Praktische Philosophie bezeichnet wird, so erleben wir heute eine Differenzierung zwischen theoretischer oder Crundlagenethik und angewandter Ethik. Damit verändert sich auch der Charakter dessen, was sich Ethik nennt.

Aus wissenssoziologischer Sicht steht außer Frage, dass die klassische Universität als Institution mit ihren Strukturen und ihrem gesellschaftlichen Auftrag die Inhalte, Methoden und somit auch Ergebnisse ihrer Disziplinen wesentlich beeinflusst. So hat die Medizinethik erhebliche Vorteile durch ihre Etablierung an den medizinischen Fakultäten erlangt. Mit der Akademisierung der Pflegeberufe erhalten auch diese eine Chance, ihre berufsspezifischen Perspektiven und Problemstellungen in ethische Diskurse einzubringen. Beiden Disziplinen ist jedoch von vornherein die professionsspezifische Perspektive mitgegeben, so dass sie stets auch für berufspolitische Interessen genutzt werden. (s.a. Kap. 2)

\subsection{Wie „Ethik“ heute in Kliniken genutzt wird}

Moderne Kliniken und Klinikunternehmen nutzen „Ethik“ in unterschiedlicher Ausformung aus verschiedensten Anlässen und zu verschiedenen Zwecken. Ethik muss dabei vom ärztlichen und pflegerischen Ethos unterschieden werden, der als Haltung und Wertekanon des Helfens und Heilens mehr immanent vorhanden ist und sich oft erst bei Verletzungen der Grundsätze explizit bemerkbar macht.

Gleichwohl stützt sich die medizinische Ethik auf das traditionelle Ethos, denn die ethische „Reflexion der Moral“ ist kein wertfreier Selbstzweck, sondern letztlich eingebettet in das Projekt einer humanen Praxis. Ethik in Medizin und Krankenhaus dient ja ausdrücklich der Klärung und Förderung dessen, was „gute Medizin“ sein soll.

Ethik im heutigen Krankenhaus und Gesundheitswesen entspricht nicht den Standards der akademischen Philosophie oder Theologie. Oft handelt es sich um Versuche, durch Anleihen an diese geisteswissenschaftlichen Disziplinen konkrete Probleme praktisch besser in den Griff zu bekommen. Das betrifft Fragen der „richtigen“ Therapieentscheidung, wenn es kein klares „richtig“ gibt. Es betrifft Fragen der Forschung an Menschen und therapeutischer Versuche. Und es betrifft Fragen der Unternehmensziele, der Organisation, der Leistungen und Angebote sowie der Allokation knapper Mittel. Nicht alles was das Prädikat „ethisch“ beansprucht muss diesem Anspruch auch gerecht sein, und oftmals wird „ethisch“ gedacht, argumentiert und entschieden, ohne dass an „Ethik“ gedacht wird. Unterscheiden muss man also auch zwischen bewusstem Einsatz ethischer Kompetenzen und Mittel und eher spontanem, intuitivem Vorge- 
hen, wenn man moralische Phänomene mehr oder weniger aus dem Abstand betrachtet und gegeneinander abwägt.

\subsubsection{Profilbildung - Marken und Unternehmensstrategie}

Krankenhäuser im Wettbewerb müssen um Patienten und Mitarbeiter konkurrieren. Im Ansehen der Bevölkerung spielen die Qualität der Versorgung und die repräsentierten Werte eine wichtige Rolle. „Ethik“ kann dabei als Markenzeichen für die Profilbildung zentral sein. Dies gilt nicht nur für konfessionelle Krankenhäuser. Ethik als Element einer spezifischen „Marke“ bedeutet, dass die ethische Kompetenz von Mitarbeitern am Krankenbett und im Management verlässlich ist und dass das gesamte Unternehmen sein Werteprofil in sein Qualitätsversprechen integriert.

Bei der Entwicklung einer prägnanten Unternehmensstrategie und der Profilierung im Sinne von Unverwechselbarkeit geht es um die Festlegung von Zielen, die man für „gut“ und „spezifisch“ hält, es geht um Prioritäten, Rangfolgen der Wichtigkeit, die Einschätzung des Gewichts von Werten usw. Auch die Wahl der Mittel, um die gewünschten Ziele zu erreichen, kann Teil eines Markenprofils sein, beispielsweise im Sinne von Fairness oder Gerechtigkeit oder in Bezug auf die Mission des Trägers. Die Prozesse zur Bildung von Entscheidungen mögen dabei rationale und intuitive Zutaten haben, stets werden sie begleitet von Bewertungen moralischer Natur. Wie soll beispielsweise die Höhe einer angestrebten Rendite festgelegt werden? Wie sollen gewonnene Gewinne eingesetzt und verteilt werden? Wie sollen die Interessen der verschiedenen Interessengruppen („Stakeholder“) gegeneinander abgewogen werden? Sollen die Gewinnziele durch Steigerung der Fallzahlen erreicht werden? Wenn ja, wie darf dieses Ziel verfolgt werden und wie nicht?

In den meisten Fällen wird bei solchen Fragen noch keine explizit ethische Kompetenz eingebracht werden. Dies kann schon deshalb nicht sein, weil der Anteil der Führungskräfte in Management und Medizin mit einer Ethik-Ausbildung oder Fortbildung in Deutschland extrem gering ist. Dies beginnt sich aber zu verändern seitdem die Betriebswirtschaftslehre zunehmend die Bedeutung moralischer Phänomene und deren ethische Reflexion in Forschung und Lehre integriert. Konfessionelle Krankenhausträger haben jedoch ihre Nähe zur theologisch geprägten Ethik früher genutzt um sich gegenüber anderen Kliniken zu profilieren. Für sie ist die „Ethik-Marke“ schon länger ein Ziel.

Es sind also auch betriebswirtschaftliche und wettbewerbsspezifische Erfordernisse die „Ethik-Strukturen“ entstehen lassen und das Management beschäftigen. „Ethik“ wird zur Ausprägung spezifischer Unternehmens- 
profile herangezogen, die als Markenzeichen die Besonderheit des Unternehmens betonen sollen. Ethik-Kodizes dienen als Ausdruck der Unternehmensidentität („Corporate Identity“) oder als Grundlage für Aktivitäten im Sinne von Unternehmensverantwortung („Corporate Social Responsibility, CSR). Im Marketing, der Öffentlichkeitsarbeit und speziell im Rahmen von Zertifizierungen sind Ethik- Kenntnisse und Kompetenzen von Vorteil. Schließlich sind Grundsätze von Fairness und Gerechtigkeit von großer Bedeutung bei Personal- und Allokationsentscheidungen sowie bei der Bewältigung von Konfliktsituationen (s. Abb. 2). Der Umgang mit Menschen verschiedener Kulturen, Sprachen und Religionen birgt darüber hinaus besonders sensible ethische Herausforderungen.
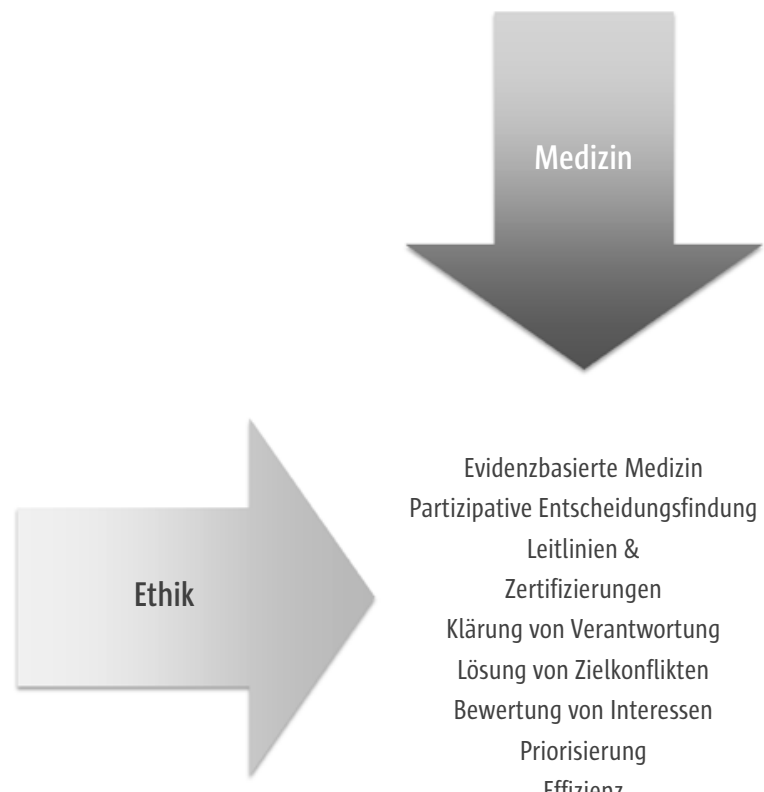

Evidenzbasierte Medizin Partizipative Entscheidungsfindung Leitlinien \& Zertifizierungen Klärung von Verantwortung Lösung von Zielkonflikten Bewertung von Interessen Priorisierung Effizienz

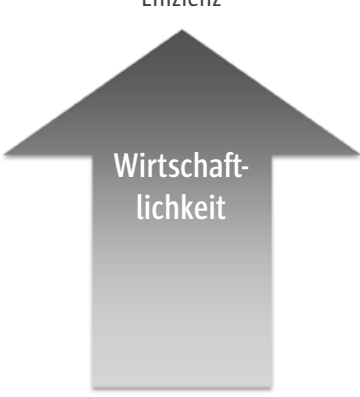

Qualität

Abb. 2 Ethik und Qualität als Instrumente einer guten Medizin 


\begin{abstract}
Ethik als „Marke" bedeutet, dass die ethische Kompetenz von Mitarbeitern am Krankenbett und im Management verlässlich ist und dass das gesamte Unternehmen sein Werteprofil in sein Qualitätsversprechen integriert.
\end{abstract}

\title{
1.7.2 Klinische Ethik für bessere Entscheidungen und zur Stärkung der Teams
}

Es ist kein Zufall, dass es die beiden großen konfessionellen deutschen Krankenhausverbände waren, die 1997 ihren Kliniken die Einführung von „Klinischen Ethik-Komitees“ empfahlen. Diese wiederum nahmen Anleihen in den USA, wo die „Hospital Ethics Commission“ schon eine etablierte Institution war und wo es bereits akademische Ausbildungsstätten und Forschungseinrichtungen für „Bioethics“, „Medical Ethics“ und „Nursing Ethics“ gab (s. Kap. 7). Die Stellungnahme dieser Krankenhausverbände zielt zwar auf die ethische Beratung bei klinischen Entscheidungen ab, es wird aber auch ausdrücklich an die gesamte Kultur des Krankenhauses gedacht, die durch „Ethik“ gefördert werden soll.

Für Führungskräfte und nachgeordnete Mitarbeiter ist es von Vorteil, wenn gelernt wurde, moralische Phänomene zu erkennen, ihre Bedeutung für Motivation und Demotivation, Kooperation oder Konflikt in der Mitarbeiterschaft zu verstehen und ihre Rolle bei der Bildung oder Zerstörung von Vertrauen bei Patienten, Kunden und Mitarbeitern zu berücksichtigen. Wenn es gelingt, die sehr häufig moralisierten und konfliktträchtigen Phänomene „mit Abstand“ zu betrachten und die eigenen Wertmaßstäbe und Entscheidungskriterien reflektiert einzubringen, ist für die Bildung von Entscheidungen viel gewonnen. Dann kann auch von echter Führung gesprochen werden, weil es eher gelingt, zwischen kontroversen Positionen Wertschätzung und Kompromissbereitschaft und damit Zusammenarbeit herzustellen.

\section{Ethical Grand Round}

Im Klinikum der Georgetown University (Washington DC) ist die „Ethical Grand Round“ eine feste Institution. Hier treffen sich zu einer stets gleichen Zeit einmal pro Woche Klinikmitarbeiter um unter Ethik-Moderation Konflikte zu besprechen, die durch kontrovers bewertete Entscheidungen ausgelöst wurden. So kann beispielsweise die Frage, wie man mit einem klar geäußerten Patientenwillen umgeht, wenn dieser absolut nicht den Vorstellungen medizinischer oder pflegerischer Standards entspricht und für den Patienten den Tod bedeutet, die Mitarbeiterschaft spalten und zu ernsthaften Belastungen des Teams einschließlich seiner Zusammenarbeit führen. Indem sich die kontroversen Be- 
wertungen unter Einschluss ihrer emotionalen Begleiterscheinungen aus neutraler Position darstellen können und unter Nutzung ethischer Theoriebildung kritisch gegeneinander abgewogen werden, wird die Kontroverse „zivilisiert“. Für die aktuellen und künftigen Entscheidungen kann dies sehr hilfreich sein, für das persönliche Befinden der Entscheider und ihrer Widersacher kann es Erleichterung bringen, die „Kultur“ der Teams gewinnt an Qualität.

In Deutschland haben mittlerweile einige Kliniken ähnlich gute Erfahrungen mit diesem Ethik-Modell gemacht, sowohl bei medizinischen Kontroversen als auch bei Konflikten organisatorischer Art. Viele Einrichtungen setzen jedoch in Unkenntnis der Unterschiede zwischen Ethik und Psychologie ausschließlich auf psychologische Moderation.

\subsubsection{Ethik-Infrastrukturen für Krankenbett und Management}

In Deutschland beginnen Aufbau und Anwendung von Ethik-Infrastrukturen durch neuartige Herausforderungen klinischer Entscheidungen, die auf Basis eines oft nur diffus formulierbaren ärztlichen Ethos allein nicht gefällt werden können. Hier liegen besonders komplexe oder neuartige Problemlagen vor, in denen nicht wirklich klar ist, worin denn das angestrebte Patientenwohl besteht. Im Vordergrund stehen hier besonders so genannte „end-of-life-decisions“, also beispielsweise Fragen der Fortsetzung, Vorenthaltung oder Beendigung lebenserhaltender Maßnahmen. Die Entwicklung der Intensivmedizin für Erwachsene Neugeborene und Kinder einerseits und die Stärkung von Patientenrechten auf Selbstbestimmung andererseits haben hier einen deutlichen Bedarf an klinischer Ethikberatung erzeugt.

Das Krankenhausmanagement scheint auf den ersten Blick nicht in derartige Entscheidungsprobleme involviert zu sein. Tatsächlich konnte in den ersten Jahren der klinischen Ethik-Komitees immer wieder beobachtet werden, dass sich nur einige Ärzte und Pflegekräfte, ggf. noch Klinikseelsorger angesprochen fühlten, an den Sitzungen teilzunehmen. Angehörige der Verwaltungs- und Managementfunktionen fühlten sich hingegen in der Regel gar nicht angesprochen. In einigen Häusern wollten die Chefärzte im Ethik-Komitee auch explizit unter sich bleiben und haben weitere Berufsgruppen aktiv ausgeschlossen.

Dennoch musste in der Regel der Impuls zur Integration von Ethik-Strukturen von den Vorständen kommen, seien sie nun ärztlicher, pflegerischer oder kaufmännischer Art. Die Leitungsebene muss das Klinische EthikKomitee wollen, muss es durch Beschluss etablieren, muss für die Freistellung der Teilnehmer, die Logistik und die Finanzierung sorgen. Für die laufende Arbeit der Komitees muss zudem die Verbindung zu den Lei- 
tungsebenen gepflegt werden, weil diese nicht selten in die Lösung verhandelter Konflikte einbezogen werden muss.

Ein kluges Klinikmanagement wird Ethik-Strukturen und Kompetenzen aus mehreren weiteren Gründen fördern. Neben der Funktion der Beratung und Entscheidungsunterstützung bei medizinischen und pflegerischen Fragen kann eine Prävention von Konflikten zwischen Mitarbeitern, Abteilungen und Berufsgruppen für die Funktionsfähigkeit ganzer Bereiche der Kliniken von hoher Bedeutung sein. Konflikte beispielweise zwischen leitenden Ärzten können nicht nur Patienten gefährden, sie stören die Arbeitsprozesse, können das Ansehen des Hauses schädigen und die Gesundheit der Mitarbeiter beeinträchtigen. Das Management muss ein Interesse an einem gesunden Arbeitsklima im Hause haben.

\section{Bedarf für ein Klinisches Ethik-Komitee?}

In einer deutschen Universitätsklinik wurde bei einem Kleinkind eine anatomische Anomalität der großen Blutgefäße am Herzen diagnostiziert. Die Operation brachte keinen Erfolg. Das Kind lief blau an, sobald es selbständig atmen musste. Es wurden weitere Operationen durchgeführt. Das Kind war dadurch über sehr viele Monate Dauerpatient auf der Intensivstation. Eine weitere Operation wurde als "letzte Chance" angesetzt. Als nach der Operation der Beatmungstubus entfernt wurde um zu prüfen, ob das Kind nun selbständig atmen kann, war das gesamte Behandlungsteam anwesend. Das Kind war bei Bewusstsein, da es ja atmen sollte. Die Atmung war erneut nicht ausreichend. Das Kind lief blau an und rang nach Luft. Einer der Ärzte wollte es erneut intubieren. Da hielten sich die Ärzte gegenseitig fest, um dem Impuls zur erneuten künstlichen Beatmung zu widerstehen. Denn nun hätte das Kind im weiteren Leben nur mit künstlicher Beatmung leben können. Das Kind erstickte vor ihren Augen. Die ärztlichen und pflegerischen Mitarbeiter, die die Szene erlebt hatten, waren dauerhaft traumatisiert. Als sie im Rahmen von Interviews befragt wurden, erzählten alle drei Ärzte und vier Pflegekräfte, dass für sie seitdem „eine dunkle Wolke“ über der Abteilung schweben würde. Der verantwortliche Oberarzt gab an, sich unmittelbar danach betrunken zu haben. Andere Mitarbeiter hatten gekündigt. Von Ärzten anderer Abteilungen kamen heftige Vorwürfe.

Wäre dieser Fall in einem klinischen Ethik-Komitee vorbereitet worden, so hätte man sich auf die entstandene Situation besser einstellen können. Wahrscheinlich hätte man sich für eine weitere Intubation und künstliche Beatmung entschieden. Das Kind wäre nicht qualvoll erstickt. Die Mitarbeiter wären nicht dauerhaft durch Schuldgefühle und Zweifel geschädigt. Klinische Ethik hätte hier auch eine psychohygienische Funktion erfüllt und vielleicht auch eine existenzielle Entlastung herbeigeführt. 
Ein wichtiger Grund für die Einbeziehung auch des kaufmännischen Managements in klinische Ethik liegt darin, dass mittels ethischer Strukturen und Kompetenzen zwar komplexe Probleme beschrieben und analysiert werden können, dass die Lösung dieser Probleme aber keineswegs durch Philosophie und Theologie erfolgen muss und kann, sondern oft auch durch Änderungen in der Organisation. Verletzungen ethischer Grundsätze der Medizin und Pflege erfolgen häufig nicht, weil den Beteiligten diese Regeln nicht bekannt wären. Sie sind häufig durch Defizite der Organisation oder der Führung bedingt.

\begin{abstract}
Es waren aufsehenerregende, häufig auch bislang unbekannte Entscheidungszwänge, hervorgerufen durch neuartige Handlungs- und Behandlungsmöglichkeiten wie die Wiederbelebungspraxis in der Intensivmedizin oder die Transplantationen mit den Problematiken des Hirntods, der Organentnahme und Organverteilung, denen die Medizin- und Bioethik ihren Aufschwung in der zweiten Hälfte des 20. Jahrhunderts verdankt. Hinzu kamen echte oder vermeintliche Skandale um Arzneimittelversuche, Zwangsbehandlungen oder wagemutige medizinische Experimente. In Deutschland war es der Fall des „Erlanger Babys“, in dem erstmals Medizinethiker in das Blickfeld der medialen Öffentlichkeit gerieten. Hier ging es um die Begründung, warum der Körper einer nach einem Verkehrsunfall hirntoten schwangeren Frau bis zur Überlebensfähigkeit des Fötus künstlich vital gehalten wurde. Regionale Aufmerksamkeit entstand für ein Klinikum, das wegen eines vermeintlich allzu groben Umgangs mit randalierenden jugendlichen „Komatrinkern“ einem organisierten Sturm von Internet-Schmähungen ausgesetzt war. Ein anderes Klinikum musste sich legitimieren, warum ein Patient sich frei im Klinikum bewegen durfte, nachdem man inn einige Tage vermisst und danach tot in einer stillgelegten Station aufgefunden hatte.
\end{abstract}

Oft sind es besondere Anlässe, in denen „Ethik“ auf den Plan gerufen wird. In solchen Fällen kann ein Klinikum ins Licht der breiten Öffentlichkeit geraten und muss zum Schutz seines Ansehens getroffene Entscheidungen moralisch legitimieren. Ein ethisch geschultes Management und eine funktionierende Ethik-Infrastruktur können dazu beitragen, die oft komplexen Hintergründe überzeugend darzustellen und gegenüber der Öffentlichkeit zu vermitteln. Auch hierfür sind also Ethik-Kompetenzen und Elemente einer Ethik-Infrastruktur hilfreich.

\title{
1.7.4 Die Ethik der Gesundheitsberufe als Vertrauensschutz
}

Jeder Führungskraft im Gesundheitswesen und speziell im Krankenhaus muss die jeweilige Kultur der verschiedenen Berufsgruppen bekannt sein. 
Von zentraler Bedeutung ist dabei der ethische Berufskodex der Ärzte, der über Jahrtausende im Kern gleichgeblieben ist. Der Weltärztebund pflegt ethische Traditionen im Geiste des „Hippokratischen Eides“, aktualisiert im „Genfer Ärztegelöbnis“ oder der „Deklaration von Helsinki“ zur Regulation der medizinischen Forschung am Menschen. Auch der Weltverband der Pflegenden (International Council of Nurses) verfügt über einen Ethikcode, ebenso Berufsverbände der Psychologen. In der Praxis ist das Wissen um diese Ethik-Kodizes jedoch lange vernachlässigt worden, so dass vielfach Unkenntnis und Unsicherheit über die Verbindlichkeit besteht.

Die Bedeutung der berufsethischen Richtlinien oder „Prinzipien“ liegt nicht darin, dass hier ein elitäres Sonderrecht einer sozial privilegierten Berufsgruppe beansprucht wird, sondern sie liegt in erster Linie im Vertrauensschutz. Ethische Codes formulieren letztlich, was die Bevölkerung von der jeweiligen Profession an Tugenden und Werten erwartet, um sich vertrauensvoll an sie wenden zu können. Am Beispiel der ärztlichen Schweigepflicht ist dies leicht nachvollziehbar.

\section{Die Ethik der Gesundheitsberufe dient dem Vertrauensschutz für Patienten und Bevölkerung.}

Damit wird ein weiterer entscheidender Aspekt deutlich: Ethik dient der Qualitätssicherung und der Patientensicherheit, und zwar schon viele Jahrhunderte bevor diese Begriffe im modernen Gesundheitswesen erfunden wurden. Fatal ist nur, wenn aus Unkenntnis dieses Faktums die heutigen Praktiken des Qualitätsmanagements „die Ethik“ bestenfalls dem „Ethik- Komitee“ oder der „Ethik-Kommission“ überlassen, und umgekehrt viele dieser Komitees und Kommissionen sich nicht zuständig fühlen für die Organisation der Medizin im Rahmen konkreter Unternehmensstrukturen.

Wozu wenn nicht zur Sicherheit und zum Schutz des Patienten dienen die traditionellen medizinethischen Grundsätze? Deren oberstes Prinzip ist traditionell die Orientierung aller Medizin, aller ärztlichen Entscheidungen und Handlungen und damit auch der medizinischen Veranstaltung „Krankenhaus“ am „Wohl des Patienten“. Die „Patient im Mittelpunkt“Parole des betriebswirtschaftlichen Managements wird hingegen von vielen Ärzten, Therapeuten und Pflegenden zynisch kommentiert, wenn ihnen die Einhaltung dieser Selbstverständlichkeit schwergemacht wird. 


\subsection{Ethik der Organisation}

Wenn die Achtung medizinethischer Grundsätze für den Vertrauensvorschuss des Patienten gegenüber dem Arzt essenziell ist, dann muss auch das Krankenhaus denselben Vertrauensschutz garantieren. Das Management kann nicht anders als medizinethische Grundsätze auch in der Organisation zu verankern. Es erfüllt diese Aufgabe besser, wenn diese nicht nur diffus gespürt werden, sondern explizit klar und entsprechend verbindlich sind.

Medizinethik und Pflegeethik werden erweitert zur Organisationsethik, wenn Medizin und Pflege in hochorganisierten Institutionen und Unternehmen praktiziert werden. Da zweifellos die Rahmenbedingungen der medizinischen Versorgung, insbesondere die Finanzierungsordnung durch das Gesundheitssystem und die Trägerstrukturen (Eigentümer) auf die konkreten Prozesse der Patientenversorgung einwirken muss die Organisationsverantwortung der Führungskräfte bzw. des Managements einbezogen werden. Damit wird der Ethikdiskurs jedoch besonders brisant und kompliziert, weil zwei unterschiedliche Orientierungen aufeinanderstoßen, die sich nicht selbstverständlich ergänzen: Ärzte und Pflegende sind darauf gepolt, die Interessen ihrer Patienten zu vertreten, das Management repräsentiert das Unternehmensinteresse und indirekt auch Interessen der Bevölkerung an einem bezahlbaren Gesundheitssystem (weil es die politisch gesetzten finanziellen Rahmenbedingungen beachten muss). Aus all dem ergibt sich, dass Organisationsethik nicht an einzelne Sonderabteilungen delegiert wird. Sie muss zur kompetenten Grundmatrix aller Führungskräfte gehören.

Gleichwohl braucht „Ethik“ im Krankenhaus und in anderen Institutionen des Gesundheitswesens strukturelle Verankerungen. Mehrere deutsche Kliniken haben diese im Rahmen von „Ethik-Projekten“ entwickelt, die ihrerseits bereits vorhandene Modelle übernommen und weiterentwickelt haben.

\subsection{Bedarf für ein Ethik-Management}

Ethik ist eine zentrale Dimension der Qualität im Krankenhaus. Wenn der Anspruch nach bestmöglicher Qualität besteht, muss die Ethik der Organisation und der Patientenversorgung aktiv organisiert und verbessert werden. 
„Ethik-Management“ ist also mehr als eine in moralischer Hinsicht „saubere“ Führungstätigkeit. Es umfasst Wissen, Kompetenzen und formelle wie informelle Strukturen. Es umfasst die Pflege der Unternehmenskultur und Aspekte der Kommunikation innerhalb des Klinikunternehmens und nach außen gegenüber der Öffentlichkeit und den Medien. In Zeiten rascher Veränderungen der Medizin, des Gesundheitssystems und der Ansprüche der Patienten und Bürger ist es ein wesentliches Element der Vertrauensbildung und auch der Profilierung im Wettbewerb, sowohl um Patienten als auch um Fachkräfte. In diesem Sinne darf die Ethik-Kompetenz des Managements und die Ethik-abhängige Unternehmenskultur nicht dem Zufall überlassen werden. Es bedarf reflektierter und gezielter Maßnahmen des Managements, um Ethik im Krankenhaus sinnstiftend und wertschaffend zu entwickeln und so zum nachhaltigen Erfolg der Organisation Krankenhaus beizutragen.

\section{Weiterführende Literatur}

Blickle G (Hrsg.) (1998) Ethik in Organisationen. Verlag für Angewandte Psychologie Göttingen

CAREUM-Stiftung (2011) Eine neue globale Initiative zur Reform der Ausbildung von Gesundheitsfachleuten. Zürich. URL: www.careum.ch/lancet-report (abgerufen am 19.04.2017)

Debatin JF, Ekkernkamp A, Schulte B, Tecklenburg A (Hrsg.) (2017) Krankenhausmanagement: Strategien, Konzepte, Methoden 3. Auflage. Medizinisch Wissenschaftliche Verlagsgesellschaft Berlin

Hellmann G (Hrsg.) (2015) Markenzeichen Ethik! Führung durch Ethik und Identität. medhochzwei Heidelberg/ Neckar

Sinek S (2011) Start with Why: How Great Leaders Inspire Everyone to Take Action (Reprint). Portfolio London

\section{Zitierte literatur}

Francis R (2013) Report of the Mid Staffordshire NHS Foundation Trust Public Inquiry, QC. The Stationery Office London 